\title{
Research on the knowledge sharing obstacles and countermeasures about teaching management in colleges and universities
}

\author{
Li Zhao ${ }^{1, a}$, Ganli Xiao ${ }^{2, b}$ \\ ${ }^{1}$ Office of Academic Affairs, Hong He University , Mengzi, China \\ 2InternationalCollege, Honghe University, Mengzi, China \\ a79836154@qq.com, b594637400@qq.com \\ 1,a Li Zhao
}

\begin{abstract}
This document expounds the concept, characteristics and significance of knowledge sharing about teaching management in colleges and universities. In five aspects, clouding knowledge, ability, psychology, resources and technology, organization, this paper analyzes the obstacle factors of knowledge sharing. Through the analysis, the paper from the management level and the administrator level puts forward some Suggestions to promote knowledge sharing about teaching management in colleges and universities.
\end{abstract}

Keywords: teaching management, knowledge sharing, obstacles, countermeasures.

\section{高校教学管理中的知识共享障碍与对策研究}

\author{
赵黎 $1, \mathrm{a}$, 肖洤澧 $2, \mathrm{~b}$ \\ 1红河学院教务处, 蒙自, 云南, 中国 \\ 2红河学院国际学院, 蒙自, 云南, 中国 \\ a79836154@qq.com, ${ }_{1, \mathrm{a}}^{5} 594637400 @ q q . c o m$ \\ $1, a_{\text {赵黎 }}$
}

中文摘要. 文章阐述了高校教学管理中的 知识共享的概念、特点及意义。分别从知识、 能力、心理、资源技术、组织五个视角对阻 碍高教教学管理知识共享的因素进行了分 析, 通过分析从高校教学管理层面和教学管 理人员层面提出了促进高校教学管理知识 共享的建议。

关键词：教学管理, 知识共享, 障碍, 对策

一、高校教学管理中的知识共享内涵 近年来, 随着高校教学改革的不断深 入, 高校教学管理改革也随之开展并逐步深
化。“现代管理的突出特点是强调人本管理 和知识管理, 即在必要的时刻把相关的信息 与知识传送给必要的人员, 使其能够有效地 利用这些信息与知识创造价值、增加效益。” 从这个意义上说, 知识共享可以在解决高校 教学管理队伍素质不高、管理观念老化、管 理方法单一、核心管理知识的流失等现实问 题上提供有效帮助, 可以促进高校教学管理 队伍素质提升和提高高校教学管理水平的 重要途径。

高校教学管理中的知识共享是指高校 的教学管理人员通过有关技术和手段将自 己的知识贡献给其他教学管理者, 并在与其 他教学管理者的交流中把别人贡献出来的 
对自己有用的知识为我所用, 从而达到促进 高校教学管理者素养, 提高高校教学管理水 平的目的, 它包括了对贡献知识和吸收知识 两个要素。高校教学管理中的知识共享首先 具有非盈利的特性, 即表现为共享的知识不 能以营利为目的; 其次, 高校教学管理中的 知识共享具有选择性, 即在高校教学管理 中, 知识共享的内容并非随意共享, 而是需 要有选择的进行知识共享, 要选择有利于提 升高校教学管理者素养, 有利于提高高校教 学管理水平的知识进行共享, 特别是那些管 理中的有效经验、空门或技术等核心知识的 共享; 再次, 高校教学管理中的知识共享具 有明确目的性, 即总是指向促进高校教学管 理者素养全员提高和部门教学管理水平提 升。

\section{二、高校教学管理中的知识共享障碍类型 (一) 知识障碍}

1、知识量障碍

在高校教学管理中, 管理知识的数量和 质量是导致知识共享能否开展和有效开展 的前提和基础。从目前高校教学管理人员队 伍研究来看, 教学管理人员管理理念滞后、 队伍素养不高是比较突出的两个问题, 这就 决定了高校教学管理人员对促进教学管理 者素养和提高教学管理水平方面的知识持 有的数量不足, 质量不高, 从而, 导致在知 识共享活动中可共享知识量不足, 共享水平 不高。

\section{2、知识特性障碍}

稀缺性是知识的一个重要特征, 包括相 对稀缺和绝对稀缺两类。“对于需要某方面 知识而这些知识偏偏又没有附着于自身的 那部分群体或个人来说, 这些知识是缺乏 的, 我们就可以称这类知识相对于这部分群 体或个体而言处于相对稀缺的状态, 而对于 目前人类探索实践中还没能发掘的那部分 知识而言, 这些未被发掘的知识则处于绝对 稀缺状态, 正是知识的稀缺性促使人们不断 的探索发现, 不断的学习交流; ”在知识共 享活动中, 由于某些教学管理知识稀缺, 导 致了知识共享的困难; 同时, 知识具有隐性 特点, 就是知识往往与知识所有者的个性、 经验、信仰、所处情景等相联系, 表现出复
杂性、不易表达和交流等特性, 这些高度个 体化的知识, 难以学习、储存和传播, 从而 也容易造成共享的困难。

3、知识成本障碍

知识成本是知识经济下出现的概念, 知 识成本包含有知识直接生产成本、交流成本 与消费成本。当知识成本较高时, 教学管理 者即便有共享的意愿, 但是也会因为知识成 本较高而放弃共享。

\section{（二）能力障碍}

1、知识洞察障碍

洞察就是能敏锐、迅速、透彻的观察和 抓住问题要害和本质, 高校教学管理活动中 的知识共享前提之一是对可以开展共享的 知识需要有敏锐的观察力和准确的判断力, 需要洞察到哪些管理知识可以共享, 哪些管 理知识共享会更利于教学管理水平的提升 等。但是在实际的教学管理中, 很多教学管 理人员在共享知识的洞察方面比较薄弱, 以 至于不知道自己可以共享什么知识和获取 什么知识。

2、知识表达障碍

英国物理化学家和哲学家波兰尼 (Michael Polanyi) 将知识分为显性知识 和隐性知识, 认为 “人有两种类型的知识。 通常称作知识的是以书面文字、图表和数学 公式加以表达的知识, 只是其中的一种类 型。没有被表达的知识是另一种知识, 比如 我们在做某件事情的行动中所掌握的知 识”。知识共享需要先将知识表征化、显性 化, 但是在高校的教学管理实际中, 由于教 学管理队伍知识显性化能力不足, 导致很多 核心管理知识不能用口头、书面形式加以表 达，致使很多核心管理知识没有被共享。

3、反思能力障碍

反思, 是对自身的审视和分析过程, 高 校教学管理活动需要反思的参与。反思可以 让高校教学管理人员跳出管理本身, 从更高 的视角来审视、分析自身在教学管理工作中 存在的问题或获得的重要经验等, 有利于促 进教学管理人员自我提高和教学管理工作 的改进, 但是在现实的高校教学管理中, 管 理人员往往因为教学管理事务的繁杂而失 去了反思的时间, 也因为自身水平的限制缺 乏反思的能力, 或反思能力低下。 


\section{（三）心理障碍}

知识共享的主体是人, 由于高校中各个 教学管理岗位的管理人员在年龄、个性特 征、价值取向等方面各不相同, 导致了在教 学管理中的知识共享活动一定程度上取决 于教学管理人员的认识和态度。阻碍高校教 学管理中知识共享主要有利已型心理和惰 性型心理两类。

1、利已型心理障碍

在高校教学管理活动中存在职位升迁、 职称晋升、评优评奖等, 而这些活动往往和 管理人员自身素养、工作绩效等有较直接的 关系。知识共享核心在于将附着于管理者个 人的对教学管理工作有促进和提升作用的 管理知识的共享, 这些知识一旦共享出来, 极有可能在同事中失去自身优势, 因此, 为 了自身利益, 管理者宁愿选择知识的不共 享。

\section{2、惰性型心理障碍}

惰性型心理的表现形式主要有自卑心 理、依赖心理、从众心理。自卑心理主要有 两种表现, 一种是认为自己经验不足, 没有 什么可以共享的知识, 另一种是羞于共享自 己的知识。高校教学管理队伍目前还存在参 差不齐的现象, 有一部分管理者对知识的洞 察能力和占有能力比较有限, 特别是一些长 期从事程序性教学管理活动的管理者, 往往 思想比较僵化, 习惯于定势思维, 很少对自 身工作有思考和创新, 所以当提及知识共享 的时候, 通常认为自己没有可以共享的知识 或者羞于共享; 依赖心理表现为在知识共享 中只希望依赖于别人进行知识共享, 自己不 积极不主动; 从众心理表现为 “人云亦云”, 自己共享的知识和别人共享的知识没有太 大其别, 导致知识共享的重复和低效。

\section{（四）资源和技术障碍}

高校教学管理中知识共享的资源和技 术障碍主要来自两方面, 一方面是在共享中 对资源和技术的投入障碍, 另一方面是对资 源和技术使用本身的障碍。资源和技术是知 识共享的基础和载体, 在信息高速发展的时 代, 资源极大丰富, 知识共享已经突破了现 场会议、面对面交谈、书面总结等这种传统 的知识共享模式, 多元的共享途径已经形
成, 利用微博、微信、 $Q Q$ 、云技术等高科技 进行知识共享已经成为可能, 但在实际的高 校教学管理中, 在引入丰富资源和各项科技 为知识共享提供支持方面还比较薄弱。同 时, 由于受高校管理队伍结构和人员管理水 平限制, 对在信息时代产生的可用于共享的 技术手段缺乏使用能力, 目前知识共享的方 式还是较多局限于单一和直接的知识共享 方式。

\section{（五）组织障碍}

\section{1、组织机构设置障碍}

萨维奇博士把管理分为五代, 第一代是 以工业时代初期以所有制为核心的管理; 第 二代是以严格等级制为特点的管理, 第三代 是以矩阵型组织为特征的管理。第四代是以 计算机网络为特点的管理, 第五代就是以知 识为核心的管理。可见，当今社会是知识管 理的社会。在教育领域, 随着企业界知识管 理的兴起和成功案例的涌现, 开展知识管 理、促进知识共享开始被越来越多的学者关 注。现行的学校教学管理组织机构中, 多为 金字塔式的科层管理结构, 多层级会导致知 识流通速度变慢, 流通渠道不畅, 会造成知 识共享低水平重复。同时, 从研究上看, 针 对知识管理和知识共享的有关职位高校还 没有设立, 知识管理和知识共享目前还处于 理念提出阶段, 由于缺乏专人管理, 还没有 真正落到行动上。

2、组织制度障碍

制度是规范人们行为的办事规程和行 动准则, 高校教学管理中的知识共享行为需 要制度支持。但是, 在现实的高校教学管理 中, 知识共享的制度寥岺无几。虽然教学管 理例会等会议中也会有知识的共享, 但是这 样的共享机会不多, 渠道单一, 而且知识共 享还往往被工作任务布置等事务性内容替 代。

\section{3、组织共享氛围障碍}

高校教学管理队伍的素质决定了知识 共享在教学管理活动中的定位永远出于不 被人重视的角落, 甚至管理人员根本就不理 解什么是知识共享, 也不知道怎样进行知识 共享, 针对高校教学管理方面的知识共享从 物质到制度到精神的共享氛围均没有形成, 
高校教学管理者中管理知识共享意愿不足 或缺失。

\section{三、促进高校教学管理中的知识共享对策建 议}

\section{（一）高校教学管理层面}

1、重视高校教学管理过程中的知识共享行 为

知识共享是知识经济时代的重要产物, 高校作为传承人类文明的重要场所, 理应紧 跟时代的步伐。高校教学管理水平直接或间 接影响学校教学活动的开展, 重视高校教学 管理队伍素质提升、有效防止高校教学管理 活动中重要管理知识的流失, 提升高校教学 管理的水平和深化管理育人的效用都是高 校教学管理层需要积极思考的问题, 而解决 这一系列问题较有实效的方法之一就是在 高校教学管理过程中开展知识的共享活动。 高校教学管理层领导要重视知识共享对高 校教学管理活动的促进作用, 普及知识管理 理念, 积极为开展知识共享提供人力、物力 和财力支持, 尽可能提供丰富的知识获取资 源和降低知识货的成本, 并用榜样的力量带 动教学管理各部门的知识共享活动, 调动全 员知识共享意愿，营造共享氛围。

2、调整建构利于高校教学管理知识共享的 组织结构

“世界上成功的企业大都进行了组织 结构的变革, 而且成效显著。如通用电气公 司 (CE) 在总裁韦尔奇上任短短的 10 多年里, 组织等级层次平均减少了 4 个, 是将近原来 的一半。根据日本公司和美国公司的统计, 实施参与式管理可以大大提高企业经济效 益, 一般都可以提高50\%以上, 有的甚至可 以提高一倍至几倍” 高校教学管理部门要尽 可能通过减少管理层次, 压缩职能机构, 裁 减人员而建立扁平化的高校教学管理组织 结构, 在条件允许的情况下, 制定专人负责 开展知识管理及共享工作。

3、提供高校教学管理知识共享制度支持

制度是开展高校教学管理知识共享活 动的有力保证, 在高校教学管理活动中, 尽 可能建立起高校教学管理知识学习和积累 制度、高校教学管理知识内部共享制度、高 校教学管理知识共享能力培训制度、高校教
学管理知识共享激励制度、师徒制度等, 通 过制度规范教学管理人员的行为规范, 促成 知识的共享, 可以有效降低因惰性心理、利 己心理和组织制度不合理造成的知识共享 障碍。

4、搭建高校教学管理知识共享平台

共享时间、共享空间、共享资源和共享 工具是知识共享平台搭建的重要基础, 提供 足够的时间和空间让高校教学管理人员进 行学习反思和进行管理知识显性化是知识 共享的前提之一。同时, 学校要积极利用现 代信息技术搭建立体和多元的分享交流平 台, 提供分享交流的策略方法指导和共享工 具的使用指导, 尽可能做到教学管理人员随 时、随地、随性都能开展知识的共享。

5、养成高校教学管理人员之间的信任关系

里斯特 (M cA 11ister，1995)将信任 分为情感基础信任和认知基础信任。情感基 础信任是指双方建立了密切的情感联系, 相 信对方的行为不会伤害自己的利益, 从而给 予的信任。认知基础信任是根据所收集到的 信息, 对对方信任的可信性做出推断后, 给 予的信任。从有关学者的研究中也得到证实 “情感信任对隐性知识共享的正向影响更 显著, 认知信任对显性知识共享的正向影响 更显著”建立在感情基础的信任更能激发高 校教学管理核心知识知识共享, 管理者之间 往往通立 “好朋友”、“亲人”、“家人” 等感情关系开展核心教学管理知识共享。信 任是高校教学管理知识共享的润滑剂, 高校 教学管理部门领导要力图通过会议、培训、 工会活动、科研活动、茶花厅、活动室等形 式搭建交流平台, 加强教学管理人员间的接 触, 尽可能促成教学管理人员间信任的养成 来达成知识共享。

\section{（二）高校教学管理人员层面}

1、增加知识积累, 开展终身学习

知识习得和积累积累是知识共享的前 提, 高校教学管理部门管理人员要重视教学 管理知识的不间断获得, 知识的习得方式和 途径可主要采取自我主动的学习、参加学校 组织的培训、进修等形式开展。高校教学管 理人员要树立终身学习的理念, 自觉开展利 于提升自身素养和提升管理能力和水平的 有关知识的学习。 


\section{2、端正共享态度}

高校教学管理人员要重新审视和看待 知识共享的价值, 认识到知识共享给教学管 理部门和自身素质提高带来的便捷, 认识到 更好的共享是为了更好的学习和工作, 主动 端正对知识共享的态度和认识, 克服知识共 享中的利已和惰性心理。

3、依托现代技术主动开展知识共享

随着科技发展, 能够用于实现知识分享 的现代技术和工具越来越多, 高校教学管理 人员要主动更新观念, 接触、学习和利用现 代化的工具和技术实现知识交流和分享, 主 动提升自己利用现代讯息技术进行知识分 享活动的技术和水平。

\section{4、提升知识共享能力}

提升知识共享的能力是高校教学管理 知识实现共享的重要环节。高校教学管理部 门人员开展知识共享活动至少需要具备三 种能力: 表达能力、可共享知识洞察能力、 反思能力。作为高校教学管理人员, 在积极 配合学校开展知识共享的前提下, 要自觉通 过各种途径提高知识共享的能力和水平。

\section{致谢}

本文为红河学院科研博硕基金项目《高 校教学管理中知识共享机制研究》 (项目编 号10BSS221）的阶段性成果之一。

\section{References}

[1] Zhang Min, On Construction Of the Teaching Management in Universities Base On BPR, Journal of Zhangjiakou Vocational and Technical College,vol.6,pp.35-36,2010.

[2] Zhao Li, A Research on the Knowledge Sharing of Teachers, master's degree thesis of East China normal university,2010.

[3] Xu Wai, Try to talk about the cost of knowledge and its compensation mode, Finance and Economics, vol.6,pp.43-47,2003.

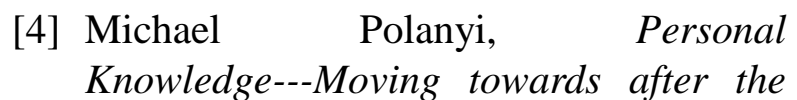

criticism, (Xu ZeminTrans.). Guizhou people press,2002,pp.95.

[5] Yi Lingfeng \& Zhu Qijing, Knowledge management, $F u$ dan university press, 2008,pp.31.

[6] Yang Yi, The Realization of Knowledge Sharing and Knowledge Innovation in an Enterprise, Information Science, vol.10,pp.1108-1109,2003.

[7] QinHongxia\&Ding Changqing, Studies on Trust Mechanism for Enterpris e Knowledge Sharing, Journal of Intelligence, vol.11,pp.43-45,2007.

[8] Chu Haonan \& Liao Jianqiao, Cognitive and emotional trust: an empirical study of influence on knowledge sharing, Science and Technology Management Res earch, vol.9,pp.238-240,2008. 